\title{
Coordination of symbiosis and cell cycle functions in Sinorhizobium meliloti
}

2

3 Shuanghong Xue, Emanuele G. Biondi*

4 Aix Marseille University, CNRS, IMM, LCB, 13009, Marseille, France.

5

$6 *$ Corresponding author: ebiondi@imm.cnrs.fr

\section{Abstract}

11 The symbiotic nitrogen fixing species Sinorhizobium meliloti represents a remarkable

12 model system for the class Alphaproteobacteria, which includes genera such as

13 Caulobacter, Agrobacterium and Brucella. It is capable of living free in the soil, and is also

14 able to establish a complex symbiosis with leguminous plants, during which its cell cycle program is completely rewired presumably due, at least in part, to the action of peptides secreted by the plant. Here we will discuss how the cell cycle regulation works in $S$. meliloti and the kinds of molecular mechanisms that take place during the infection. We will focus on the complex regulation of the master regulator of the $S$. meliloti cell cycle, the response regulator CtrA, discussing its implication in symbiosis.

21 Keywords:

22 Sinorhizobium meliloti, cell cycle, symbiosis, nitrogen fixation 
Sinorhizobium meliloti belongs to the alpha class of the Gram-negative proteobacteria (Alphaproteobacteria). It has been studied for a long time for its ability to infect roots of leguminous plants, such as those of the genus Medicago (M. sativa and M. truncatula). By a complex mechanism (that we will describe in more details in the next sections), this bacterium is able to multiply inside plant cells, within which the bacterial cells fix atmospheric nitrogen into ammonium that can be utilized by plants. In exchange, the plant provides a nutrient rich environment in which a small part of the $S$. meliloti population can benefit. The bacterial form that is able to fix nitrogen is referred to as a bacteroid. The formation of a bacteroid involves a massive differentiation program that results in a cell unable to divide, and is therefore referred to as terminally differentiated. From an evolutionary point of view, this terminal differentiation has puzzled scientists, as it is difficult to explain what is the selective advantage for the bacterium, in the context of a beneficial symbiosis (mutual exchange of nutrients), if the majority of the population is unable to replicate. In this review, a few elements will be described in order to clarify the possible evolutionary hypotheses about the role of bacteroid differentiation.

The first contact between bacteria and plants relies on a specific exchange of molecules, Nod factors produced by bacteria and the flavonoids secreted into the rhizophere (the environment that surrounds roots) by the plants (Cooper 2007; Liu and Murray 2016). The entry of the bacteria into the plant tissue occurs following the formation of a modified radical root hair, which is specifically modulated by the bacterial Nod factors (Shaw and Long 2003; Sieberer et al. 2005). This root hair then traps a few S. meliloti cells, which then penetrate inside the root tissue and induce the formation of an 
infection thread that is sealed after the entrance of few bacteria (Jones and Walker 2008). In this tunnel, bacteria divide and eventually reach the internal tissue that will host the future bacteroids. Although the mechanism is still poorly known, bacteria are introduced into the plant cell by invagination of the plant cell membrane, resulting in the bacterium being surrounded by a plant-derived membrane. This prokaryotic cell surrounded by the plant membrane is called a "symbiosome" (Jones et al. 2007). The presence of three membranes surrounding a bacteroid that are actively involved in secreting and importing nutrients raises important questions about mechanisms of transport, which up to now have been only partially explored.

As mentioned before, $S$. meliloti lives in the soil as free-living organism even without the presence of legumes (Carelli et al. 2000). This suggests that the capability to establish a symbiosis is not an essential function of the species, as revealed by the discovery of $S$. meliloti strains unable to infect plants. A recent discovery has highlighted that $S$. meliloti colonizes the plant as an endophyte, and can be recovered from leaves and other tissues (Pini et al. 2012). This discovery opened an interesting scenario about the bacteroid formation. In fact, if $S$. meliloti is able to colonize the whole plant, then the plant may have evolved a way to induce a terminal differentiation therefore blocking bacteria duplication and preventing uncontrolled colonization of the plant. As we will see in the next sections, induction of bacteroid differentiation is indeed under the control of plant signals, more specifically peptides, which are indeed able to induce bacteroid-like formation even in laboratory culture (Mergaert et al. 2006). As bacteroids are terminally differentiated, symbiotic peptides are indeed antimicrobial molecules (Kereszt et al. 2011). 


\section{Cell cycle, symbiotic infection and differentiation}

S. meliloti division produces two different cell types (Figure 1). A "small" cell that is characterized by a smaller size and the incapacity to replicate the DNA and divide (Collier 2012). The "large" cell, on the contrary, is bigger and is able to replicate its genome once per cell division. To our knowledge, there is no exception to one single round of genome replication in S. meliloti in free-living cells, as the origin of replication is strictly controlled by multiple regulatory mechanisms that ensure this perfect coordination between DNA replication and cell division (Sibley et al. 2006). Its location, adjacent to hemE, is the same as for oriC in Caulobacter crescentus, the model organism in which the origin of replication has been characterized the most among chromosomes of alphaproteobacterial origins.

The process of infection of $S$. meliloti in the plant root and multiplication inside plant cells is still poorly understood. Many functions are involved in this process, including bacterial cell cycle regulation (see next section) and specific signaling molecules produced by Medicago plants (Pan and Wang 2017). In this section we will briefly see how S. meliloti establishes this symbiosis. We will also discuss how plants control bacteroid differentiation and what possible bacterial functions may be involved.

A S. meliloti bacteroid is a special cell type (Figure 1) that possesses two important features: it is able to fix nitrogen, and it shows a clear, irreversible cell cycle arrest that is responsible for its inability to generate new cells once the nodule enters into a senescent state (Kereszt et al. 2011). Therefore, the bacteroid is metabolically active but indeed terminally differentiated (Barsch et al. 2006). The bacteroid metabolism is under the control of specific regulators named Fix and Nif that will not be discussed here (Jones et al. 2007). Morphologically, S. meliloti bacteroids are large and elongated cells (10 times bigger) with respect to the free-living cell, and therefore have a bigger 
cytoplasmic volume. Bacteroids show a certain degree of branching with cells named Yshaped having the form of the letter Y. Y-shaped cells are usually considered as bacteroids in a more mature state. A striking feature of bacteroids is the genome endoreduplication (up to 24 genome copies) (Mergaert et al. 2006), while in free-living S. meliloti, cells either have one copy of the genome or two right before the daughter cell separation (De Nisco et al. 2014). Finally the bacterial bacteroid membrane is highly permeable, suggesting a strong exchange of molecules with the host plant cell (Mergaert et al. 2006).

Bacteroid differentiation is controlled by peptides produced by plants, more than 600 in M. truncatula (Mergaert et al. 2003), called nodule-specific cysteine rich (NCR) peptides, but only a few of them have been characterized in detail. Indeed, the majority of this large family of peptides has only been predicted by bioinformatics based on the $M$. truncatula genome, while the existence of only 138 of them has been experimentally confirmed (Durgo et al. 2015). However, even for the most characterized peptides, our knowledge is still very preliminary, and the mechanism of action of those peptides on the bacterial cell is still far from understood.

One of the most investigated peptides is called NCR247, which is produced by $M$. truncatula (Van de Velde et al. 2010). Several studies have shown that this NCR peptide may affect multiple targets and functions. NCR247 is able to induce a certain degree of bacteroid differentiation in free-living bacteria cultivated in lab conditions (Van de Velde et al. 2010; Penterman et al. 2014; Farkas et al. 2014). Cysteine residues of NCR247 can be modified by di-sulfur bridge formation changing its targets with respect to the redox state (Haag et al. 2012; Shabab et al. 2016). NCR247 can penetrate the bacterial membrane and form complexes with several bacterial proteins (Farkas et al. 2014). For example this peptide interacts with FtsZ presumably inhibiting bacterial cell 
123 division (Farkas et al. 2014). It also interacts with ribosomal proteins affecting 124 translation and altering the proteome and the physiology of the endosymbiont. NCR247 125 is further able to directly interact with the chaperone GroEL, which is required for 126 efficient infection, terminal differentiation and nitrogen fixation. Of more interesting 127 from the perspective of this review is the link between NCR247 and the cell cycle 128 regulators of $S$. meliloti (as we will explore in more details in the next section). In 129 particular, sub-lethal doses of NCR247 are able to induce a cell cycle defect similar to 130 bacteroids, by specifically affecting regulons of two master regulators of the cell cycle: 131 GcrA and CtrA (Penterman et al. 2014). The first regulator was discovered and 132 characterized in C. crescentus (Holtzendorff et al. 2006; Fioravanti et al. 2012; Murray et 133 al. 2013; Mohapatra et al. 2014), while in S. meliloti its role is linked to cell cycle but its 134 mode of action is still unknown (Robledo et al. 2015). On the contrary, CtrA has been 135 studied in S. meliloti in greater detail. CtrA plays a clear role as the master regulator of 136 cell cycle, as we will see in the next sections, suggesting that a peptide directly or 137 indirectly acting on its regulon would influence cell differentiation (Pini et al. 2015).

138 Although NCR247 shows a clear negative antimicrobial effect in vitro, and it's 139 presumably implicated in the differentiation of bacteroids, other peptides may actually 140 play a protective role in the plant tissues. This is the case, for example, for NCR169 and 141 NCR211, which were localized in the cellular space between the bacterial membrane 142 and the plant membrane of the symbiosome (Horváth et al. 2015; Kim et al. 2015).

143 Finally, at least one membrane transporter is important for the activity of the NCR 144 peptides, BacA (Marlow et al. 2009; Haag et al. 2011). More specifically, BacA is able to 145 internalize several antimicrobial peptides and it's necessary to protect the cells to 146 peptide treatments in plants (Haag et al. 2011). The presence of a transporter involved 147 in the peptides activity suggests that the targets of the peptides should be also located in 
148 the bacterial cytoplasm. Recently a genetic screening for transposon mutants resistant 149 to NCR247 revealed that tens of genes may protect cells from this peptide (Arnold et al. 150 2017). Those genes are mostly involved in membrane, peptidoglycan and cell envelope 151 physiology, but are also associated with internal functions such as regulation of 152 transcription factors or factors associated with ribosomes. These discoveries suggest 153 that the activity of each peptide may be very general acting on many levels, and possibly 154 involved in rewiring the whole physiology of the bacterial cell. 155 
159 Regulation of cell cycle in alphaproteobacterial species, such as S. meliloti, C. crescentus, 160 Agrobacterium tumefaciens or Brucella abortus, is based on several conserved factors, 161 called master regulators, that regulate most of the genes controlling essential steps in 162 cell cycle progression. Although our knowledge is still preliminary in many bacterial 163 models, it is reasonable to say that the master regulators CtrA, DnaA, GcrA and CcrM are 164 well-conserved cell cycle factors in most of the species of the class Alphaproteobacteria 165 (Wright et al. 1997; Barnett et al. 2001; Brilli et al. 2010). DnaA is a conserved helicase that regulates the initiation of DNA replication in bacteria (Sibley et al. 2006; Skarstad and Katayama 2013). Removing its binding sites in the origin of replication results in a complete arrest of DNA replication (Sibley et al. 2006). As revealed by a bioinformatic analysis of alphaproteobacterial genomes, almost all factors that regulate the cell cycle in the model system C. crescentus are also present in S. meliloti (Brilli et al. 2010). The 171 exceptions will be commented in the next paragraphs of this section. This conservation suggests a common evolution of the cell cycle program in the two organisms. However, 173 as we will specifically discuss for S. meliloti, every alphaproteobacterial species appears 174 different from the others, suggesting that the cell cycle machinery has diverged in every 175 species in order to adapt to different life styles and physiologies (Brilli et al. 2010).

176 As previously mentioned, the response regulator of the family of two-component 177 systems, named CtrA (Cell cycle Transcriptional Regulator A), plays a crucial role in the 178 regulation of the cell cycle in alphaproteobacterial species as demonstrated for the first 179 time in the model species C. crescentus (Quon et al. 1996). Response regulators are 180 generally proteins composed by a receiver domain (REC), with a conserved aspartic 181 residue, and an output domain, which usually binds DNA. Phosphorylation of the REC 
182

183

184

185

186

domain usually leads to dimerization (Gao and Stock 2009), creating an active dimer of the response regulator that is able to bind its consensus sequence at the promoter region of target genes, thereby regulating the genes' expression. CtrA presumably belongs to this class of response regulators, suggesting that a dimeric form of phosphorylated CtrA should interact with its palindromic consensus sequence that we can approximate to AATT(N7)AATT. This consensus sequence is conserved across alphaproteobacterial species, from Rickettsia to Caulobacter, Sinorhizobium, Magnetospirillum or Rhodobacter (Brassinga et al. 2002; Brilli et al. 2010; Mercer et al. 2010; Greene et al. 2012). Based on the presence of this consensus in the promoter region of genes of alphaproteobacterial genomes, the conservation of functions in all species was analyzed in silico, revealing that CtrA in all species belonging to this alphaproteobacterial class is usually linked to motility, which is probably the ancestral function controlled by CtrA (Greene et al. 2012; Mercer et al. 2012). In species belonging to the Caulobacterales (C. crescentus) and Rhizobiales (S. meliloti, B. abortus and A. tumerfaciens, for example), CtrA potentially controls, in addition to motility, cell cyclerelated functions such as cell division and DNA methylation (Brilli et al. 2010). This link to essential functions, such as cell division, explains the essential nature of the $\operatorname{ctr} A$ gene in those species, while in species in which CtrA controls only motility, the disruption of the gene only affects the flagellum biogenesis and possibly other non-essential functions (Greene et al. 2012; Mercer et al. 2012).

A combination of Chromatin Immunoprecipitation-deep sequencing (ChIPseq) and transcriptomic analysis in S. meliloti revealed the direct and non-direct regulons of CtrA (Pini et al. 2015). Although many genes are annotated as hypothetical, and require further characterization, several functions appeared to be clearly controlled by CtrA. 
Several motility and chemotaxis genes are indirect targets of CtrA, such as genes encoding the flagellum apparatus of S. meliloti (i.e. flgBCDH and fliEFIL).

Among the important cell cycle regulators that will be introduced in the next paragraphs, the genes sciP and $\operatorname{div} J$ are directly controlled by $\mathrm{Ctr} A$ in $S$. meliloti. Unlike in C. crescentus, CtrA indirectly regulates divK transcription in S. meliloti, while CtrA also

211 regulates the second DivK-kinase encoding $\operatorname{cbrA}$ expression in $S$. meliloti but not in $C$. 212 crescentus, where cbrA is not present. So this alternative architecture may give a 213 differential degree of control in the negative feedback loop regulating CtrA functions in 214 S. meliloti.

215 Genes $\min C$ and $\min D$ are the only characterized cell division-related genes directly 216 repressed by CtrA in $S$. meliloti. In $S$. meliloti, as with many other bacteria, MinC and 217 MinD repress cell division by inhibiting FtsZ polymerization and Z-ring formation in the 218 polar regions (Shih and Zheng 2013).

219 In C. crescentus and S. meliloti, CtrA indeed controls DNA replication and cell division; 220 DNA replication is negatively regulated by CtrA, while cell division genes are directly 221 and positively activated by CtrA (Pini et al. 2015). This dual and opposite activity 222 suggests that CtrA levels and activity must change during the cell cycle; at the onset of 223 DNA replication, CtrA must be inactive in order to activate DNA replication, while in the 224 following steps, CtrA must be present in order to activate crucial functions. This 225 observation implies that CtrA activity should be highly regulated. In this section we will 226 also review all those CtrA regulatory mechanisms.

227 In C. crescentus the negative control of DNA replication is dependent on the presence of 228 CtrA binding sites at the origin of replication (Quon et al. 1998). In contrast, no CtrA 229 binding sites have been found in the DnaA-dependent DNA replication origin of $S$. 230 meliloti, suggesting either an alternative negative control or possibly the absence of this 
231 regulation (Sibley et al. 2006; Pini et al. 2015). CtrA has been further characterized in $S$.

232 meliloti, revealing that the $\operatorname{ctr} A$ gene is indeed essential for the growth of the bacterium

233 (Barnett et al. 2001; Pini et al. 2015). Although the orthologous genes of the

234 phosphorylation cascade of CtrA are present in S. meliloti (Brilli et al. 2010), their

235 characterization has never been carried out. On the contrary, the role of the CtrA-

236 inhibitor DivK, which is a single receiver domain of the two-component system protein

237 family, similar to CheY, has been intensively investigated in S. meliloti together with its

238 complex kinase/phosphatase module, composed by the kinases DivJ and CbrA and the

239 phosphatase PleC (Lam et al. 2003; Gibson et al. 2006; Gibson et al. 2007; Sadowski et al.

240 2013; Pini et al. 2013; Schallies et al. 2015). DivK, in C. crescentus, is an essential factor

241 for cell cycle progression as loss of function mutants of divK are arrested at the G1 phase

242 (Hecht et al. 1995). DivK is also essential in $S$. meliloti and acts as the main negative

243 regulator of CtrA (Pini et al. 2015). The absence of DivK, or an inability of DivK to be

244 phosphorylated, results in a stable and constitutively active CtrA that in turns blocks the

245 origin of replication.

246 DivK shows dynamic localization during cell cycle progression, as shown by GFP fusions

247 (Lam et al. 2003), and its localization depends on the polarity factor PodJ1 (Fields et al.

248 2012). The active form of DivK, responsible for CtrA inhibition, is the phosphorylated

249 form, DivK P. DivK is phosphorylated by two kinases, DivJ and CbrA (Pini et al. 2013),

250 which both contribute to the pool of DivK $\sim$ P. Deletion of either of the two kinases leads

251 to a severe cell cycle defect showing elongated and branched cells with a slow growth

252 rate. However, the double deletion of $\operatorname{divJ}$ and $c b r A$ is lethal, strongly demonstrating that

253 phosphorylation of DivK is absolutely necessary for a proper cell cycle progression (Pini

254 et al. 2013). Conversely, the ability at specific stages of the cell cycle to remove the

255 phosphate group from DivK $\sim \mathrm{P}$ is also essential as the only known DivK phosphatase, 
PleC, is indispensable in S. meliloti (Fields et al. 2012; Pini et al. 2013). Surprisingly, in $C$. crescentus deletion of DivJ, the only known DivK kinase in this species, or deletion of the DivK phosphatase PleC are possible, as is the double deletion, while mutation of the phosphorylation site in DivK is not tolerated by C. crescentus cells. This observation 260 suggests a redundant function that may compensate for the absence of DivK 261 phosphorylation (Lori et al. 2015), or an alternative phosphorylation pathway. In $S$. 262 meliloti, this redundancy is observed, arguing that different species have evolved a 263 unique architecture of the cell cycle network.

264 The expression of almost 500 genes varies as a function of the cell cycle in S. meliloti (De 265 Nisco et al. 2014). As in C. crescentus, many genes show peak expression corresponding 266 with the timing of their cellular function (De Nisco et al. 2014). This time-regulated 267 expression of genes, which are required for specific functions, was analyzed by 268 developing a new method of synchronization for $S$. meliloti, based on the induction of the 269 stringent response (carbon and nitrogen starvation) able to induce G1-blocked cells by 270 Rel-dependent ppGpp accumulation (De Nisco et al. 2014). G1-blocked cells were then 271 able to proceed through a complete and synchronized cell cycle with only one DNA 272 replication cycle, ultimately leading to an asymmetrical cell division.

273 The genome of $S$. meliloti consists of three replicons: a 4 mega-bases circular 274 chromosome with a single DnaA-dependent origin of replication, a replicon, named 275 pSymB, that contains two essential genes and many genes involved in the adaptation to 276 environmental niches, and a dispensable megaplasmid, named pSymA, mostly 277 associated with symbiosis (Galibert et al. 2001; Capela et al. 2001; Finan et al. 2001). 278 DNA replication in this organism was analyzed further by looking at the origin of 279 replication of the three large replicons of S. meliloti (Frage et al. 2016). Surprisingly the 280 three origins of replication are temporally and spatially separated in the cell, with the 
281 chromosome being the first to be replicated with its origin located very close to the 282 polar regions. The megaplasmid pSymA follows the chromosome replication with its 283 origin located in proximity of the pole but shifted towards the center of the cell. Finally 284 pSymB replication starts after pSymA and its origin localization at the beginning of its 285 replication is almost at mid-cell (Frage et al. 2016). This remarkable organization 286 suggests that DNA replication in $S$. meliloti is highly organized with replicons that are 287 kept in the right subcellular localization by mechanisms that are still unknown.

288 CtrA encoding gene transcription is driven by a complex promoter region with at least 289 two different promoters, named P1 and P2 (Barnett et al. 2001). As in C. crescentus, CtrA 290 protein levels change as a function of cell cycle, with the protein levels at a minimum 291 during the G1-S transition (initiation of the chromosome replication) (Pini et al. 2015). 292 Presumably this decrease of CtrA levels depends on a mechanism of active degradation 293 of the protein, which depends on the protease ClpXP and several alphaproteobacterial 294 proteins that are present also in C. crescentus. Specifically the single receiver domain 295 protein CpdR, active in the non-phosphorylated form, is required for CtrA degradation and symbiosis (Kobayashi et al. 2009; Pini et al. 2015; Schallies et al. 2015). Moreover 297 the protein RcdA is not dispensable in S. meliloti and it's required, as in C. crescentus, for 298 CtrA degradation, as a conditional mutant of $\operatorname{rcdA}$ shows high levels of CtrA and a lethal 299 block of cell cycle (Pini et al. 2015).

\section{Symbiosis and the cell cycle}

302 Surprisingly, the phenotype of $c t r A$ depletion resembles the morphology of bacteroids 303 with elongated and enlarged cells that sometimes showing a Y shaped form (Pini et al. 304 2015). Moreover ctrA-depleted cells also show an increase in genome ploidy as 305 bacteroids with all replicons increasing equally their copy number (Pini et al. 2015). 
306 This phenotype is consistent with the absence of the CtrA protein in bacteroids 307 extracted from nodules (Pini et al. 2013), and the observation that ctrA is barely 308 expressed in the zone of differentiation, while the DNA replication initiation factor DnaA 309 is highly expressed (Roux et al. 2014). These results are also consistent with the results 310 of plants inoculated with a $c p d R$ deletion mutant, a protein required for CtrA proteolysis.

311 The nodules $\Delta c p d R$ inoculated plants are unable to fix nitrogen and contain bacteria that 312 are not differentiated into bacteroids, consistent with a model in which cells with a 313 stable CtrA are unable to differentiate in bacteroids (Kobayashi et al. 2009). As said 314 before, NCR247-treated cells experience a down-regulation of the CtrA-controlled genes, 315 consistent with a mechanism in which bacteroid differentiation depends on CtrA 316 depletion. This results is further reinforced by the observation of a symbiotic defect of a 317 divJ deletion mutant that shows cells arrested in the intracellular infection (Pini et al. 318 2013).

319 The link between morphology of bacteroids and nitrogen fixation is not clear yet. For 320 example, the shape and membrane surface/volume ratio of bacteroids may influence the 321 nitrogen fixing performance. On the contrary, the plant's ability to induce terminal 322 differentiation could be instead linked to the necessity to produce bacterial farms, 323 unable to divide but efficiently fixing nitrogen. The latter explanation could suggest that 324 bacteroid formation is required by plants in order to avoid a dangerous multiplication of 325 bacteria inside the plant tissues. 


\section{Conclusions}

328 Regulators of bacterial cell cycle are undoubtedly involved in the bacteroid 329 differentiation program of $S$. meliloti. The regulatory network that coordinates DNA 330 replication, cell division and presumably bacteroid differentiation relies on the activity 331 of a master regulator of cell cycle named CtrA, whose role in cell cycle regulation is 332 conserved across alphaproteobacterial species, such as $C$. crescentus, B. abortus and $A$. 333 tumefaciens (Brilli et al. 2010). Among alphaproteobacteria, C. crescentus is one of the 334 best models in which cell cycle regulation has been intensively investigated. More recently other bacterial species, such as $S$. meliloti, have also been analyzed in more 336 detail, revealing that although factors are conserved, every species has a unique 337 behavior with differences that may reflect the adaptation to specific life-styles.

338 In S. meliloti, CtrA is essential for viability and controls essential functions such as cell 339 division, DNA replication and DNA methylation. Moreover it controls motility and its 340 regulation by direct activation of the expression of cell cycle regulators. For example, 341 CtrA controls its activity by regulating the DivK module directly (Div) and CbrA) and 342 indirectly (DivK itself). This negative feedback from CtrA to DivK, the inhibitor of CtrA 343 activity, is also present in C. crescentus; however, in C. crescentus, this essential 344 transcriptional feedback is directly acting on the divK gene (Biondi et al. 2006). From a systems biology point of view, the two different architectures in C. crescentus and $S$. 346 meliloti, although similar, may underline a different response to, for example, 347 environmental variations or stresses. Further investaigation on the mechanistic 348 properties of $S$. meliloti should reveal important features of this architecture.

349 An increasing body of evidence suggests that CtrA may be a crucial factor during 350 bacteroid differentiation. Its absence in mature bacteroids and the phenotype of a CtrA 
351 loss of function strongly suggest that inactivation of CtrA is an essential step in the 352 development of bacteroids. Recent evidences have also pointed that peptides, such as 353 NCR247, may be targeting directly or indirectly CtrA and its complex regulatory 354 apparatus. Research should focus now on revealing this molecular link between NCR 355 peptides and the cell cycle machinery. 
Figure 1. Schematics of $S$. meliloti cell cycle. Cells are rod-shaped and contain three replicons, here represented with three different colors. The chromosome, in red, is the biggest, pSymB is in green, and the smallest is pSymA in blue. Every cell division, two different cell types are formed: a large cell and a small cell, each containing a copy of replicon. The large cell is able to immediately initiate a new round of DNA replication (S

363 phase), while the small cell (G1) must first differentiate into a large cell. Replicons do 364 not replicate at the same time; the chromosome is the first replicon to initiate its replication, followed by pSymA and then pSymB. Moreover, the single origins of replication of each replicon are spatially localized. The chromosome origin has a polar

367 localization, the pSymA origin is proximal to the polar regions, while pSymB possesses 368 almost a mid cell localization. Molecular determinants responsible for this spatial 369 organization of the chromosome are still poorly known. Due to the secretion of NCR 370 peptides by leguminous plants such as M. sativa, S. meliloti undergoes differentiation 371 becoming larger and longer and accumulating all three replicons up to 24 copies. The 372 dotted line suggests that the connection of bacteroid differentiation and the free-living 373 cell cycle is still unknown. Bacteroid differentiation is defined "terminal" as, to our 374 knowledge, there is no possibility of cell division by bacteroids. Bacteroids are surrounded by a plant membrane (orange line).

377 Figure 2. Cell cycle regulation network in $\boldsymbol{S}$. meliloti. The circuit is centered on the 378 response regulator CtrA that regulates multiple general functions, such as motility, pilus 379 biogenesis and chemotaxis. More specifically, phosphorylated CtrA (CtrA-P) activates 380 the expression of $c c r M$, an essential methyl-transferase that regulates the cell cycle, sciP 
381 encoding the homolog of the C. crescentus inhibitor of CtrA activity, and finally DivJ and 382 CbrA, the two kinases of DivK. On the contrary, PleC functions as a phosphatase, 383 removing the phosphate from DivK-P. CtrA directly represses (solid red lines) the Min 384 system, which in turn has an inhibitory activity on FtsZ. Although the molecular link is 385 still unclear, CtrA (dotted red lines) plays a positive role on $\operatorname{divK}$ transcription and 386 presumably is essential for the coordination of DNA replication, as the absence of CtrA 387 leads to an accumulation of chromosomes. Phosphorylation of CtrA presumably requires 388 DivL, CckA and ChpT and it is inhibited by phosphorylated DivK (DivK-P). Finally CtrA 389 (and/or CtrA-P) is degraded by ClpPX-dependent proteolysis that requires two adapter 390 proteins, named RcdA and CpdR1. 


\section{Acknowledgements}

393

394 We thank members of the Biondi lab for a fruitful and stimulating discussion about cell

395 cycle regulation in alphaproteobacteria and for George DiCenzo for precious comments 396 and insightful suggestions.

397 
1. Arnold MFF, Shabab M, Penterman J, Boehme KL, Griffitts JS, Walker GC (2017) Genome-Wide Sensitivity Analysis of the Microsymbiont Sinorhizobium meliloti to Symbiotically Important, Defensin-Like Host Peptides. mBio 8: . doi: 10.1128/mBio.01060-17

2. Barnett MJ, Hung DY, Reisenauer A, Shapiro L, Long SR (2001) A homolog of the CtrA cell cycle regulator is present and essential in Sinorhizobium meliloti. J Bacteriol 183:3204-3210 . doi: 10.1128/JB.183.10.3204-3210.2001

3. Barsch A, Tellström V, Patschkowski T, Küster H, Niehaus K (2006) Metabolite profiles of nodulated alfalfa plants indicate that distinct stages of nodule organogenesis are accompanied by global physiological adaptations. Mol PlantMicrobe Interact MPMI 19:998-1013 . doi: 10.1094/MPMI-19-0998

4. Biondi EG, Reisinger SJ, Skerker JM, Arif M, Perchuk BS, Ryan KR, Laub MT (2006) Regulation of the bacterial cell cycle by an integrated genetic circuit. Nature 444:899-904 . doi: 10.1038/nature05321

5. Brassinga AKC, Siam R, McSween W, Winkler H, Wood D, Marczynski GT (2002) Conserved response regulator CtrA and IHF binding sites in the alphaproteobacteria Caulobacter crescentus and Rickettsia prowazekii chromosomal replication origins. J Bacteriol 184:5789-5799

6. Brilli M, Fondi M, Fani R, Mengoni A, Ferri L, Bazzicalupo M, Biondi EG (2010) The diversity and evolution of cell cycle regulation in alpha-proteobacteria: a comparative genomic analysis. BMC Syst Biol 4:52 . doi: 10.1186/1752-0509-452

7. Capela D, Barloy-Hubler F, Gouzy J, Bothe G, Ampe F, Batut J, Boistard P, Becker A, Boutry M, Cadieu E, Dréano S, Gloux S, Godrie T, Goffeau A, Kahn D, Kiss E, Lelaure V, Masuy D, Pohl T, Portetelle D, Pühler A, Purnelle B, Ramsperger U, Renard C, Thébault P, Vandenbol M, Weidner S, Galibert F (2001) Analysis of the chromosome sequence of the legume symbiont Sinorhizobium meliloti strain 1021. Proc Natl Acad Sci U S A 98:9877-9882 . doi: 10.1073/pnas.161294398

8. Carelli M, Gnocchi S, Fancelli S, Mengoni A, Paffetti D, Scotti C, Bazzicalupo M (2000) Genetic diversity and dynamics of Sinorhizobium meliloti populations nodulating different alfalfa cultivars in Italian soils. Appl Environ Microbiol 66:4785-4789

9. Collier J (2012) Regulation of chromosomal replication in Caulobacter crescentus. Plasmid 67:76-87 . doi: 10.1016/j.plasmid.2011.12.007

10. Cooper JE (2007) Early interactions between legumes and rhizobia: disclosing complexity in a molecular dialogue. J Appl Microbiol 103:1355-1365 . doi: 10.1111/j.1365-2672.2007.03366.x

11. De Nisco NJ, Abo RP, Wu CM, Penterman J, Walker GC (2014) Global analysis of cell cycle gene expression of the legume symbiont Sinorhizobium meliloti. Proc Natl Acad Sci U S A. doi: 10.1073/pnas.1400421111

12. Durgo H, Klement E, Hunyadi-Gulyas E, Szucs A, Kereszt A, Medzihradszky KF, Kondorosi E (2015) Identification of nodule-specific cysteine-rich plant peptides in endosymbiotic bacteria. Proteomics. doi: 10.1002/pmic.201400385

13. Farkas A, Maróti G, Durgő H, Györgypál Z, Lima RM, Medzihradszky KF, Kereszt A, 
Mergaert P, Kondorosi E (2014) Medicago truncatula symbiotic peptide NCR247 contributes to bacteroid differentiation through multiple mechanisms. Proc Natl Acad Sci U S A 111:5183-5188 . doi: 10.1073/pnas.1404169111

14. Fields AT, Navarrete CS, Zare AZ, Huang Z, Mostafavi M, Lewis JC, Rezaeihaghighi Y, Brezler BJ, Ray S, Rizzacasa AL, Barnett MJ, Long SR, Chen EJ, Chen JC (2012) The conserved polarity factor podJ1 impacts multiple cell envelope-associated functions in Sinorhizobium meliloti. Mol Microbiol 84:892-920 . doi: 10.1111/j.1365-2958.2012.08064.x

15. Finan TM, Weidner S, Wong K, Buhrmester J, Chain P, Vorhölter FJ, HernandezLucas I, Becker A, Cowie A, Gouzy J, Golding B, Pühler A (2001) The complete sequence of the 1,683-kb pSymB megaplasmid from the N2-fixing endosymbiont Sinorhizobium meliloti. Proc Natl Acad Sci U S A 98:9889-9894 . doi: 10.1073/pnas.161294698

16. Fioravanti A, Clantin B, Dewitte F, Lens Z, Verger A, Biondi EG, Villeret V (2012) Structural insights into ChpT, an essential dimeric histidine phosphotransferase regulating the cell cycle in Caulobacter crescentus. Acta Crystallograph Sect F Struct Biol Cryst Commun 68:1025-1029 . doi: 10.1107/S1744309112033064

17. Frage B, Döhlemann J, Robledo M, Lucena D, Sobetzko P, Graumann PL, Becker A (2016) Spatiotemporal choreography of chromosome and megaplasmids in the Sinorhizobium meliloti cell cycle. Mol Microbiol 100:808-823 . doi: 10.1111/mmi.13351

18. Galibert F, Finan TM, Long SR, Puhler A, Abola P, Ampe F, Barloy-Hubler F, Barnett MJ, Becker A, Boistard P, Bothe G, Boutry M, Bowser L, Buhrmester J, Cadieu E, Capela D, Chain P, Cowie A, Davis RW, Dreano S, Federspiel NA, Fisher RF, Gloux S, Godrie T, Goffeau A, Golding B, Gouzy J, Gurjal M, Hernandez-Lucas I, Hong A, Huizar L, Hyman RW, Jones T, Kahn D, Kahn ML, Kalman S, Keating DH, Kiss E, Komp C, Lelaure V, Masuy D, Palm C, Peck MC, Pohl TM, Portetelle D, Purnelle B, Ramsperger U, Surzycki R, Thebault P, Vandenbol M, Vorholter FJ, Weidner S, Wells DH, Wong K, Yeh KC, Batut J (2001) The composite genome of the legume symbiont Sinorhizobium meliloti. Science 293:668-672 . doi: 10.1126/science.1060966

19. Gao R, Stock AM (2009) Biological insights from structures of two-component proteins. Annu Rev Microbiol 63:133-154 . doi: 10.1146/annurev.micro.091208.073214

20. Gibson KE, Barnett MJ, Toman CJ, Long SR, Walker GC (2007) The symbiosis regulator CbrA modulates a complex regulatory network affecting the flagellar apparatus and cell envelope proteins. J Bacteriol 189:3591-3602 . doi: 10.1128/JB.01834-06

21. Gibson KE, Campbell GR, Lloret J, Walker GC (2006) CbrA is a stationary-phase regulator of cell surface physiology and legume symbiosis in Sinorhizobium meliloti. J Bacteriol 188:4508-4521 . doi: 10.1128/JB.01923-05

22. Greene SE, Brilli M, Biondi EG, Komeili A (2012) Analysis of the CtrA pathway in Magnetospirillum reveals an ancestral role in motility in alphaproteobacteria. J Bacteriol 194:2973-2986 . doi: 10.1128/JB.00170-12

23. Haag AF, Baloban M, Sani M, Kerscher B, Pierre O, Farkas A, Longhi R, Boncompagni E, Hérouart D, Dall'angelo S, Kondorosi E, Zanda M, Mergaert P, Ferguson GP (2011) Protection of Sinorhizobium against host cysteine-rich antimicrobial peptides is critical for symbiosis. PLoS Biol 9:e1001169 . doi: 10.1371/journal.pbio.1001169 
24. Haag AF, Kerscher B, Dall'Angelo S, Sani M, Longhi R, Baloban M, Wilson HM, Mergaert P, Zanda M, Ferguson GP (2012) Role of cysteine residues and disulfide bonds in the activity of a legume root nodule-specific, cysteine-rich peptide. J Biol Chem 287:10791-10798 . doi: 10.1074/jbc.M111.311316

25. Hecht GB, Lane T, Ohta N, Sommer JM, Newton A (1995) An essential single domain response regulator required for normal cell division and differentiation in Caulobacter crescentus. EMBO J 14:3915-3924

26. Holtzendorff J, Reinhardt J, Viollier PH (2006) Cell cycle control by oscillating regulatory proteins in Caulobacter crescentus. BioEssays News Rev Mol Cell Dev Biol 28:355-361 . doi: 10.1002/bies.20384

27. Horváth B, Domonkos Á, Kereszt A, Szűcs A, Ábrahám E, Ayaydin F, Bóka K, Chen Y, Chen R, Murray JD, Udvardi MK, Kondorosi É, Kaló P (2015) Loss of the nodulespecific cysteine rich peptide, NCR169, abolishes symbiotic nitrogen fixation in the Medicago truncatula dnf7 mutant. Proc Natl Acad Sci U S A 112:15232-15237 . doi: $10.1073 /$ pnas.1500777112

28. Jones KM, Kobayashi H, Davies BW, Taga ME, Walker GC (2007) How rhizobial symbionts invade plants: the Sinorhizobium-Medicago model. Nat Rev Microbiol 5:619-633 . doi: 10.1038/nrmicro1705

29. Jones KM, Walker GC (2008) Responses of the model legume Medicago truncatula to the rhizobial exopolysaccharide succinoglycan. Plant Signal Behav 3:888-890

30. Kereszt A, Mergaert P, Kondorosi E (2011) Bacteroid development in legume nodules: evolution of mutual benefit or of sacrificial victims? Mol Plant-Microbe Interact MPMI 24:1300-1309 . doi: 10.1094/MPMI-06-11-0152

31. Kim M, Chen Y, Xi J, Waters C, Chen R, Wang D (2015) An antimicrobial peptide essential for bacterial survival in the nitrogen-fixing symbiosis. Proc Natl Acad Sci U S A 112:15238-15243 . doi: 10.1073/pnas.1500123112

32. Kobayashi H, De Nisco NJ, Chien P, Simmons LA, Walker GC (2009) Sinorhizobium meliloti CpdR1 is critical for co-ordinating cell cycle progression and the symbiotic chronic infection. Mol Microbiol 73:586-600 . doi: 10.1111/j.1365-2958.2009.06794.x

33. Lam H, Matroule J-Y, Jacobs-Wagner C (2003) The asymmetric spatial distribution of bacterial signal transduction proteins coordinates cell cycle events. Dev Cell 5:149-159

34. Liu C-W, Murray JD (2016) The Role of Flavonoids in Nodulation Host-Range Specificity: An Update. Plants Basel Switz 5: . doi: 10.3390/plants5030033

35. Lori C, Ozaki S, Steiner S, Böhm R, Abel S, Dubey BN, Schirmer T, Hiller S, Jenal U (2015) Cyclic di-GMP acts as a cell cycle oscillator to drive chromosome replication. Nature 523:236-239 . doi: 10.1038/nature14473

36. Marlow VL, Haag AF, Kobayashi H, Fletcher V, Scocchi M, Walker GC, Ferguson GP (2009) Essential role for the BacA protein in the uptake of a truncated eukaryotic peptide in Sinorhizobium meliloti. J Bacteriol 191:1519-1527 . doi: 10.1128/JB.01661-08

37. Mercer RG, Callister SJ, Lipton MS, Pasa-Tolic L, Strnad H, Paces V, Beatty JT, Lang AS (2010) Loss of the response regulator CtrA causes pleiotropic effects on gene expression but does not affect growth phase regulation in Rhodobacter capsulatus. J Bacteriol 192:2701-2710 . doi: 10.1128/JB.00160-10

38. Mercer RG, Quinlan M, Rose AR, Noll S, Beatty JT, Lang AS (2012) Regulatory systems controlling motility and gene transfer agent production and release in Rhodobacter capsulatus. FEMS Microbiol Lett 331:53-62 . doi: 10.1111/j.1574- 
6968.2012.02553.x

39. Mergaert P, Nikovics K, Kelemen Z, Maunoury N, Vaubert D, Kondorosi A, Kondorosi E (2003) A novel family in Medicago truncatula consisting of more than 300 nodule-specific genes coding for small, secreted polypeptides with conserved cysteine motifs. Plant Physiol 132:161-173 . doi: 10.1104/pp.102.018192

40. Mergaert P, Uchiumi T, Alunni B, Evanno G, Cheron A, Catrice O, Mausset A-E, Barloy-Hubler F, Galibert F, Kondorosi A, Kondorosi E (2006) Eukaryotic control on bacterial cell cycle and differentiation in the Rhizobium-legume symbiosis. Proc Natl Acad Sci U S A 103:5230-5235 . doi: 10.1073/pnas.0600912103

41. Mohapatra SS, Fioravanti A, Biondi EG (2014) DNA methylation in Caulobacter and other Alphaproteobacteria during cell cycle progression. Trends Microbiol 22:528-535 . doi: 10.1016/j.tim.2014.05.003

42. Murray SM, Panis G, Fumeaux C, Viollier PH, Howard M (2013) Computational and genetic reduction of a cell cycle to its simplest, primordial components. PLoS Biol 11:e1001749 . doi: 10.1371/journal.pbio.1001749

43. Pan H, Wang D (2017) Nodule cysteine-rich peptides maintain a working balance during nitrogen-fixing symbiosis. Nat Plants 3:17048 . doi: 10.1038/nplants.2017.48

44. Penterman J, Abo RP, De Nisco NJ, Arnold MFF, Longhi R, Zanda M, Walker GC (2014) Host plant peptides elicit a transcriptional response to control the Sinorhizobium meliloti cell cycle during symbiosis. Proc Natl Acad Sci U S A 111:3561-3566 . doi: 10.1073/pnas.1400450111

45. Pini F, De Nisco NJ, Ferri L, Penterman J, Fioravanti A, Brilli M, Mengoni A, Bazzicalupo M, Viollier PH, Walker GC, Biondi EG (2015) Cell Cycle Control by the Master Regulator CtrA in Sinorhizobium meliloti. PLoS Genet 11:e1005232 . doi: 10.1371/journal.pgen.1005232

46. Pini F, Frage B, Ferri L, De Nisco NJ, Mohapatra SS, Taddei L, Fioravanti A, Dewitte F, Galardini M, Brilli M, Villeret V, Bazzicalupo M, Mengoni A, Walker GC, Becker A, Biondi EG (2013) The DivJ, CbrA and PleC system controls DivK phosphorylation and symbiosis in Sinorhizobium meliloti. Mol Microbiol 90:5471. doi: $10.1111 / \mathrm{mmi} .12347$

47. Pini F, Frascella A, Santopolo L, Bazzicalupo M, Biondi EG, Scotti C, Mengoni A (2012) Exploring the plant-associated bacterial communities in Medicago sativa L. BMC Microbiol 12:78 . doi: 10.1186/1471-2180-12-78

48. Quon KC, Marczynski GT, Shapiro L (1996) Cell cycle control by an essential bacterial two-component signal transduction protein. Cell 84:83-93

49. Quon KC, Yang B, Domian IJ, Shapiro L, Marczynski GT (1998) Negative control of bacterial DNA replication by a cell cycle regulatory protein that binds at the chromosome origin. Proc Natl Acad Sci U S A 95:120-125

50. Robledo M, Frage B, Wright PR, Becker A (2015) A stress-induced small RNA modulates alpha-rhizobial cell cycle progression. PLoS Genet 11:e1005153 . doi: 10.1371/journal.pgen.1005153

51. Roux B, Rodde N, Jardinaud M-F, Timmers T, Sauviac L, Cottret L, Carrère S, Sallet E, Courcelle E, Moreau S, Debellé F, Capela D, de Carvalho-Niebel F, Gouzy J, Bruand C, Gamas P (2014) An integrated analysis of plant and bacterial gene expression in symbiotic root nodules using laser-capture microdissection coupled to RNA sequencing. Plant J Cell Mol Biol 77:817-837 . doi: $10.1111 /$ tpj.12442 
52. Sadowski C, Wilson D, Schallies K, Walker G, Gibson KE (2013) The cycle regulation. Microbiol Read Engl. doi: 10.1099/mic.0.067504-0

53. Schallies KB, Sadowski C, Meng J, Chien P, Gibson KE (2015) Sinorhizobium meliloti CtrA Stability Is Regulated in a CbrA-Dependent Manner That Is Influenced by CpdR1. J Bacteriol 197:2139-2149 . doi: 10.1128/JB.02593-14

54. Shabab M, Arnold MFF, Penterman J, Wommack AJ, Bocker HT, Price PA, Griffitts JS, Nolan EM, Walker GC (2016) Disulfide cross-linking influences symbiotic activities of nodule peptide NCR247. Proc Natl Acad Sci U S A 113:10157-10162 . doi: $10.1073 /$ pnas.1610724113

55. Shaw SL, Long SR (2003) Nod factor elicits two separable calcium responses in Medicago truncatula root hair cells. Plant Physiol 131:976-984 . doi: 10.1104/pp.005546

56. Shih Y-L, Zheng M (2013) Spatial control of the cell division site by the Min system in Escherichia coli. Environ Microbiol 15:3229-3239 . doi: 10.1111/14622920.12119

57. Sibley CD, MacLellan SR, Finan T (2006) The Sinorhizobium meliloti chromosomal origin of replication. Microbiol Read Engl 152:443-455 . doi: 10.1099/mic.0.28455-0

58. Sieberer BJ, Timmers ACJ, Emons AMC (2005) Nod factors alter the microtubule cytoskeleton in Medicago truncatula root hairs to allow root hair reorientation. Mol Plant-Microbe Interact MPMI 18:1195-1204 . doi: 10.1094/MPMI-18-1195

59. Skarstad K, Katayama T (2013) Regulating DNA replication in bacteria. Cold Spring Harb Perspect Biol 5:a012922 . doi: 10.1101/cshperspect.a012922

60. Van de Velde W, Zehirov G, Szatmari A, Debreczeny M, Ishihara H, Kevei Z, Farkas A, Mikulass K, Nagy A, Tiricz H, Satiat-Jeunemaître B, Alunni B, Bourge M, Kucho K, Abe M, Kereszt A, Maroti G, Uchiumi T, Kondorosi E, Mergaert P (2010) Plant peptides govern terminal differentiation of bacteria in symbiosis. Science 327:1122-1126 . doi: 10.1126/science.1184057

61. Wright R, Stephens C, Shapiro L (1997) The CcrM DNA methyltransferase is widespread in the alpha subdivision of proteobacteria, and its essential functions are conserved in Rhizobium meliloti and Caulobacter crescentus. J Bacteriol 179:5869-5877 
Figure 1

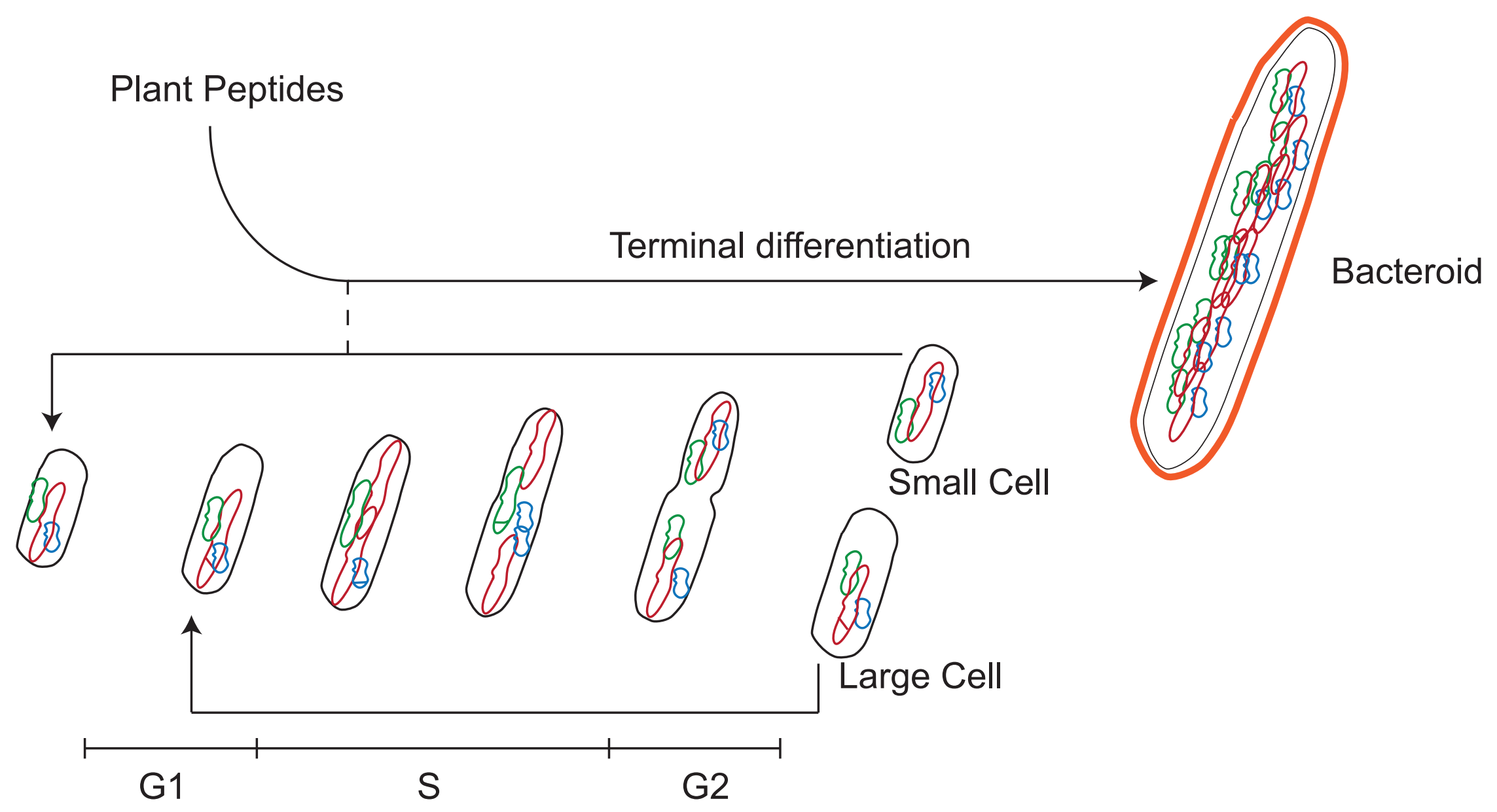


Figure 2

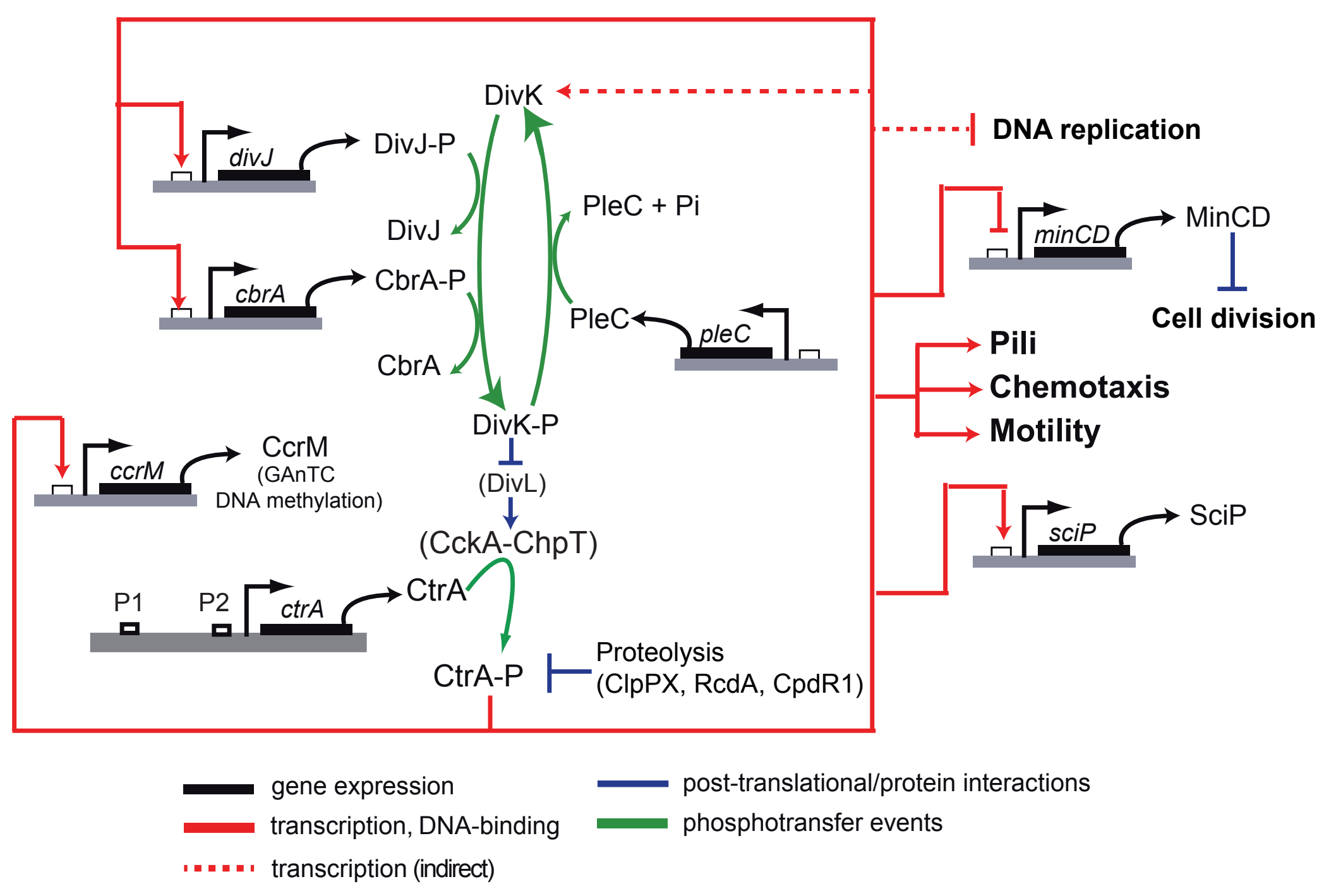

